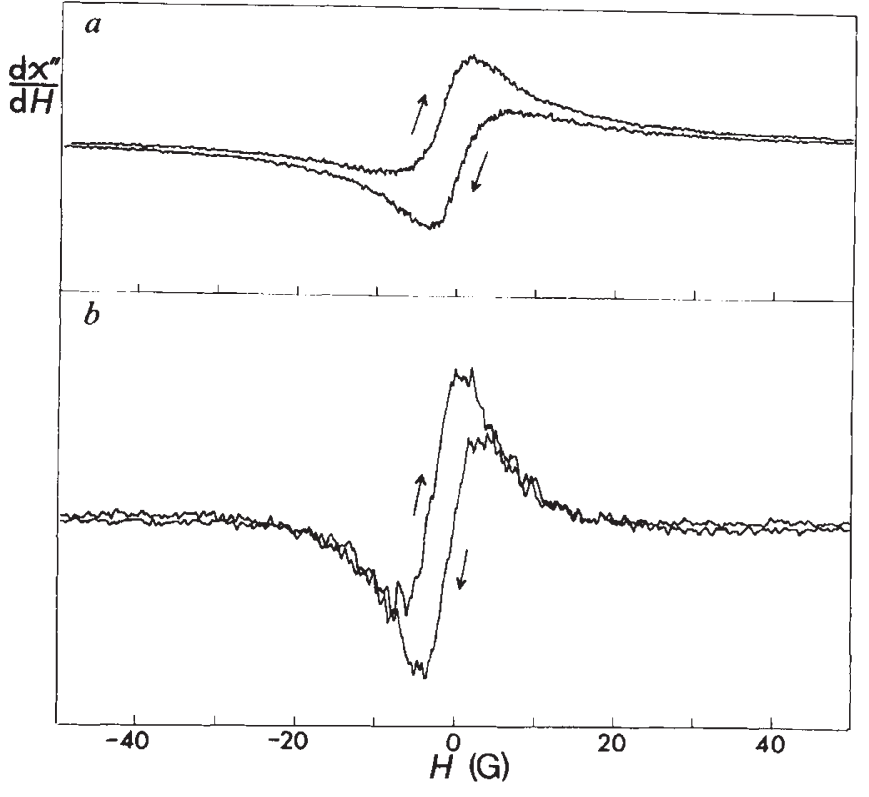

Low-field ESR spectra of $\mathrm{YBa}_{2} \mathrm{Cu}_{3} \mathrm{O}_{7-1}: a$, at $77 \mathrm{~K}$, obtained with an $\mathrm{X}$-band spectrometer (microwave frequency $9.45 \mathrm{GHz}) ; b$, at $60 \mathrm{~K}$. at S-band $(2.40 \mathrm{GHz})$. The measured signal is the field derivative of the imaginary part of the magnetic susceptibility $\left(\chi^{\prime \prime}\right)$, as a function of the external magnetic field.

spin states with a zero-field splitting of the order of $0.3 \mathrm{~cm}^{-1}$, as proposed in ref. 1 .

Insight into this non-resonant microwave absorption is obtained from one additional measurement, which demonstrates that the amplitude of the absorption is insensitive to whether the $H_{1}$ field of the microwave radiation is parallel or perpendicular to the external magentic field. These observations are consistent with the studies of Blazey et al. ${ }^{2}$ and of Khachaturyan et al. ${ }^{3}$, and support the proposed mechanism of non-resonant absorption. Blazey et al. ${ }^{2}$ used low-field microwave absorption data to argue that the material is a superconducting glass which consists of weakly coupled superconducting clusters.

T.-S. LIN

Department of Chemistry,

L.G. SOBOTKA

Washington University, St Louis,

Missouri 63130, USA

National Biomedical ESR Center,

Medical College of Wisconsin,

Milwaukee, Wisconsin 53226, USA

\title{
A conserved NTP-motif in putative helicases
}

SiR-Escherichia coli recBCD enzyme is a multifunctional protein involved in general recombination which possesses, among other functions, an ATP-dependent DNA helicase activity ${ }^{1}$. Inspection of the amino-acid sequences of the complex subunits reveals that two of them, $r e c B$ and $r e c D$, contain a consensus pattern of residues characteristic of the catalytic sites of many enzymes that use nucleoside triphosphates (NTPs), the socalled NTPmotif $^{2}$. This prompted us to perform a more detailed computer-assisted comparison of the sequences of these two proteins with those of other enzymes of this class.

This comparison reveals an unexpected similarity between $\mathrm{rec} D$ and the NTPmotif-containing domain of a non-structural protein of beet necrotic yellow vein virus (BNYVV), a positive-strand RNA plant virus. This domain belongs to a recently identified family of homologous viral proteins (domains) involved in virus RNA replications ${ }^{3.4}$. In these proteins the NTP-motif is one of the most strictly conserved stretches of sequence.

For one of them, tobacco mosaic virus (TMV) protein p126, the NTP-binding capacity has been demonstrated experi-

mentally ${ }^{5}$. The optimal alignment of the NTP-motif-containing domains of rec $D$ and of the BNYVV and TMV proteins is shown in the figure. The similarity is high enough to suggest a monophyletic origin for the compared domains (see table).

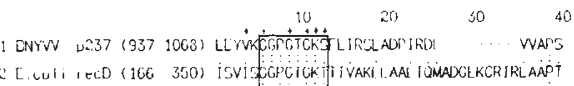

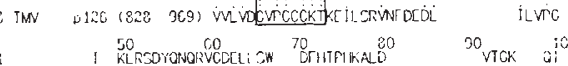

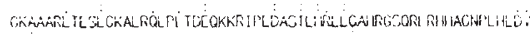

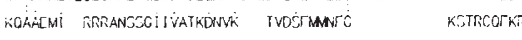

$$
\begin{aligned}
& \begin{array}{lllll}
110 & 120 & 130 & 140 \quad 150
\end{array}
\end{aligned}
$$

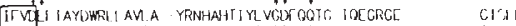

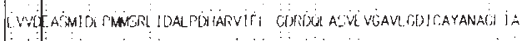

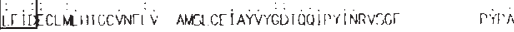

$$
\begin{aligned}
& 100100
\end{aligned}
$$

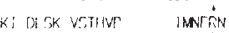

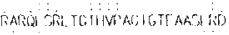

$$
\begin{aligned}
& \text { A kilv biverk kibisc }
\end{aligned}
$$

Optimal alignment of the NTP-motif-containing domains of recD and the presumptive NTPases of BNYVV and TMV. Sequences from refs 1 (rec D), 7 (BNYVV) and 8 (TMV). Alignment generated by the program OPTAL, based on the original algorithm of Sankoff". Identical residues (:) and conservative replacements (.) are highlighted. Asterisks, residues constituting the putative viral NTP-motif consensus $^{4.11}$ The residues constituting the NTPmotif proper are boxed

\begin{tabular}{|c|c|c|c|}
\hline \multicolumn{4}{|c|}{ Summary of the alignments } \\
\hline & $\operatorname{rec} D$ & BNYVV & TMV \\
\hline $\operatorname{rec} D$ & - & $28.8(51.5)$ & $16.2(35.2)$ \\
\hline BNYVV & 5.9 & - & $20.5(31.8)$ \\
\hline TMV & 3.7 & 3.2 & - \\
\hline
\end{tabular}

Below the diagonal: Alignment scores calculated in standard deviation (s.d.) units for the three alignments. Note that the similarity between the rec $D$ and BNYVV segments is the highest and exceeds the threshold of $5 \mathrm{~s}$.d. thought to be indicative of a true evolutionary relationship ${ }^{10}$. Above the diagonal: per cent similarity expressed as strict coincidence and, in parentheses, coincidence including conservative replaccments.

Strikingly, the similarity betwen the rec $D$ and BNYVV sequences is even higher than that between the two viral sequences. Such a pronounced sequence similarity could be due to conservation of a specific NTP-requiring function. Thus, viral NTP. motif-containing proteins may be subunits of RNA helicases involved in the unwinding of double-stranded replication forms during viral RNA replication, and in recombination between RNA genomes, a process recently described for plant viruses ${ }^{h}$.

Alexander E. Gorbalenya EUGENE V. KOONIN Alexel P. Donchienko VLADIMIR M. BI.INOV Institute of Poliomyelitis and Viral Encephalitides,

USSR Academy of Medical Sciences, 142782 Moscow Region, USSR

1. Finch, P.W. et al. Nucleic Acids Res. 14, 8583-8594 (1986) 2. Walker, J.E., Saraste, M., Runswick, M.J. \& Gay, N.J. EMBO J. 1. 945-951 (1982)

3. Gorbalenya, A.E., Blinov, V.M., Koonin, E.V. Molek. Genetika 11, 30-36(1985).

4. Gorbalenya, A.E. et al. Molek. Biol. 21, 1566-1571 (1987). 5. Evans, R.K., Haley, B.E. \& Roth, D.A.J. biol. Chem. 260, 7800-7804 (1985)

6. Bujarski, J.J. \& Kaesherg, P. 321, 528-531 (1986).

7. Bouzoubaa, S. et al. J. gen. Virol. 68, 615-626(1987).

8. Goelet, P. et al. Proc natn. Acad. Sci. USA 79, 5818 (1982)

9. Pozdnyakov, V.I. \& Pankov, Yu.A. Int. J. Peptide Protein Res. 17, 284-291 (1981)

10. Doolittle, R.F. Science 214, 149-159 (1981).

11. Gorbalenya, A.E., Blinov, V.M., Donchenko, A.P. \& Koonin. E.V. J. molec. Evol. (in the press).

\section{A new superfamily of replicative proteins}

SiR-I report a set of 21 related proteins, identified by computer searches, which are all involved in nucleic-acid replication and/or recombination. These include two Escherichia coli ATP-dependent helicases, four essential human herpesvirus proteins (probably also helicases), two exonuclease V (ExoV) subunits, and the yeast PIF protein (involved in mitochondrial DNA recombination). These helicases and nucleases are structurally related to a set of conserved domains (20-70\% amino-acid identity) that are common in RNA viruses ${ }^{1}$, and which I used as the starting point for the search.

The $u v r D$ and rep helicases are both 